\title{
Snap-Through and Pull-In Instabilities of an Arch-Shaped Beam Under an Electrostatic Loading
}

\author{
Yin Zhang, Yisong Wang, Zhihong Li, Member, IEEE, Yubo Huang, and Dachao Li
}

\begin{abstract}
The snap-through and pull-in instabilities of the micromachined arch-shaped beams under an electrostatic loading are studied both theoretically and experimentally. The pull-in instability that results in a system collision with an electrode substrate may lead to a system failure and, thus, limits the system maximum displacement. The beam/plate structure with a flat initial configuration under an electrostatic loading can only experience the pull-in instability. With the different arch configurations, the structure may experience either only the pull-in instability or the snap-through and pull-in instabilities together. As shown in our computation and experiment, those arch-shaped beams with the snap-through instability have the larger maximum displacement compared with the arch-shaped beams with only the pull-in stability and those with the flat initial configuration. The snapthrough occurs by exerting a fixed load, and the structure experiences a discontinuous displacement jump without consuming power. Furthermore, after the snap-through jump, the structures are demonstrated to have the capacity to withstand further electrostatic loading without pull-in. Those properties of consuming no power and increasing the structure deflection range without pull-in is very useful in microelectromechanical systems design, which can offer better sensitivity and tuning range. [2006-0232]
\end{abstract}

Index Terms-Actuators, beams, electrostatic analysis, modeling.

\section{INTRODUCTION}

$\mathbf{T}$ HE INSTABILITY study of chevron-shaped or V-shaped structures, as shown in Fig. 1(a), plays an important role in catastrophe theory [1], [2] and engineering applications [3]. The snap-through instability pattern experienced by the chevronshaped or V-shaped structures is also shared by other problems like the buckling propagation of a pipeline and a beam on a

Manuscript received October 26, 2006; revised January 25, 2007. The work of Y. Zhang was supported in part by the National Natural Science Foundation of China under Grant 10502050 and in part by the Scientific Research Foundation for the Returned Overseas Chinese Scholars, State Education Ministry of China. The work of $\mathrm{Z}$. Li was supported by the National Key Laboratory of Nano/Micro Fabrication Technology Foundation. Subject Editor S. Spearing.

Y. Zhang is with the State Key Laboratory of Nonlinear Mechanics, Institute of Mechanics, Chinese Academy of Sciences, Beijing 100080, China (e-mail: zhangyin@lnm.imech.ac.cn).

Y. Wang is with the National Key Laboratory of Nano/Micro Fabrication Technology, Institute of Microelectronics, Peking University, Beijing 100871, China (e-mail: wangys@ime.pku.edu.cn).

$\mathrm{Z}$. Li is with the MEMS Center, Institute of Microelectronics, Peking University, Beijing 100871, China (e-mail: zhhli@ime.pku.edu.cn).

Y. Huang is with the State Key Laboratory of Precision Measuring Technology and Instruments, Tianjin University, Tianjin 300072, China (e-mail: bobohyb@yahoo.com.cn).

$\mathrm{D}$. Li is with the College of Precision Instrument and Opto-Electronics Engineering, Tianjin University, Tianjin 300072, China (e-mail: dchli@ tju.edu.cn).

Color versions of one or more of the figures in this paper are available online at http://ieeexplore.iee.org.

Digital Object Identifier 10.1109/JMEMS.2007.897090

\section{(a) One DOF system snap-through}

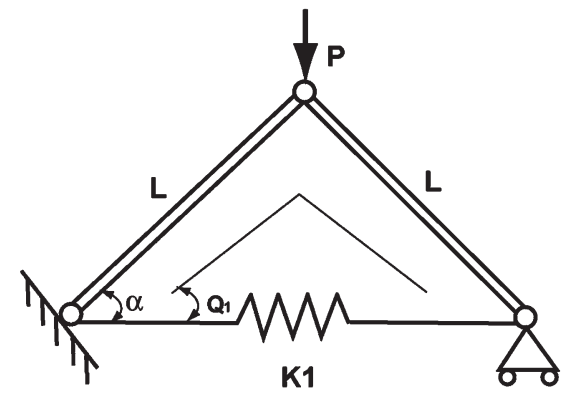

(b) One DOF system pull-in

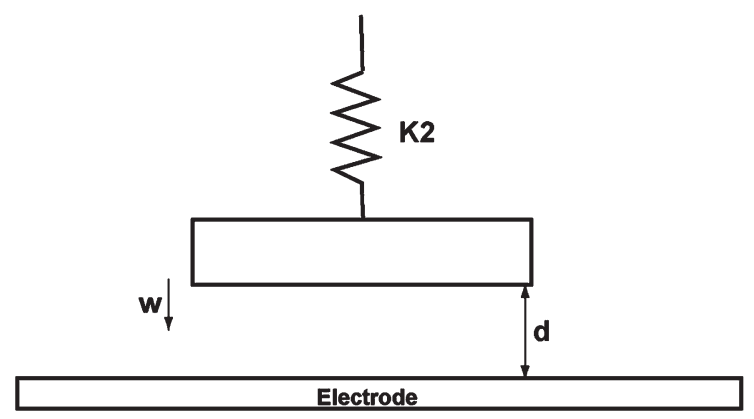

Fig. 1. Schematic diagram of one-DOF systems with the snap-through and pull-in instabilities, respectively. (a) Two trusses are tied with a spring $K_{1}$ at the bottom and under a mechanical concentrated load $P$ at the center. (b) Plate with a spring $K_{2}$ under an electrostatic loading.

nonlinear elastic foundation [4]. As shown in Fig. 2 of the snap-through of $\mathrm{V}$-shaped trusses, the rotation angle increases dramatically and so does its slope when the load is around the critical value. When the critical load is reached, the slope becomes infinite, and snap-through occurs. After the snapthrough, the slope becomes finite, and the trusses regain the capacity to withstand further loading without losing stability. Both the statics and dynamics of the arch-shaped structures are intensively studied during the 1950s and the 1960s [5]-[12]. A relatively recent experiment on an elastic arch instability done by Pippard [13] inspires the investigators again to further explore the rich instability patterns and dynamics of the archshaped structures [14], [15]. The snap-through property of the arch-shaped or V-shaped structures and the advantages like reliability, reconfigurability, and low cost have been utilized in various devices such as robotic manipulators, crane-like devices for space exploration, and electrostrictive polymer artificial muscle (EPAM) actuators [3]. When the snap-through happens, the applied force is fixed, and the structure equilibrium experiences a rather dramatic jump to a new stable one. In that sense, the structure is reconfigured by exerting a force but consuming 


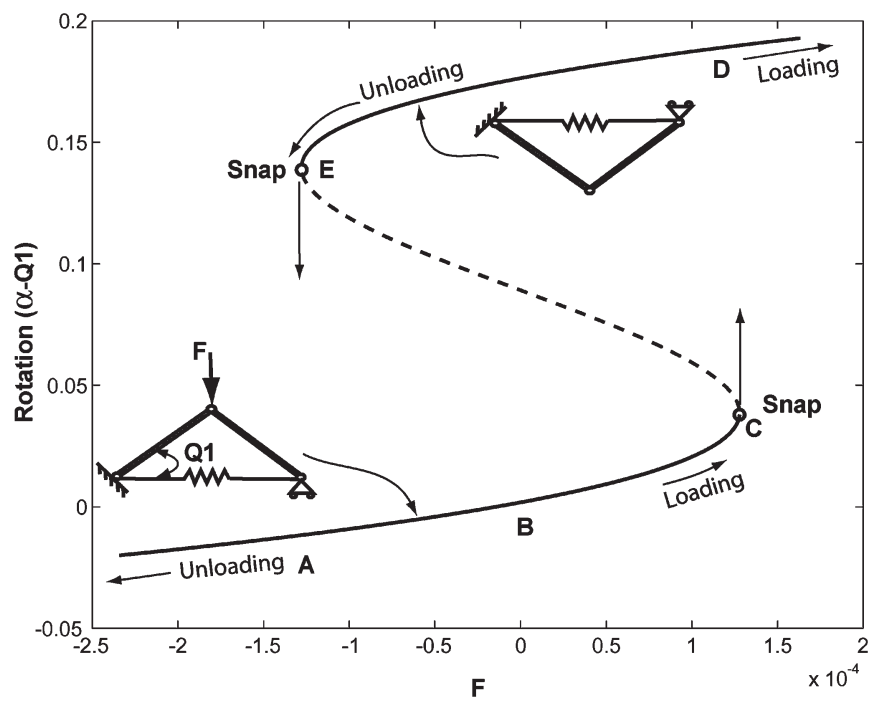

Fig. 2. Snap-through instability of V-shaped trusses under a mechanical concentrated load and the curve of $F$ versus rotation angle.

no power, and powering is not a trivial thing in the world of micromechanics [16]. Various microdevices such as actuator [16], [19]-[24], microvalve [17], and transducer [18] utilizing the snap-through instability have been reported. In microelectromechanical systems (MEMS), another instability called pull-in also plays a very important role in MEMS device performance and design [25]-[31]. Pull-in is such instability at which the system elastic restoring force can no longer balance the attractive forces such as the electrostatic [25]-[28], [31] or van der Waals (vdw) [30] forces. When pull-in happens, the MEMS structure collapses and may adhere to the structure, which results in the failure of the system. In the aforementioned archshaped or V-shaped structures, the transverse force exerted on those structures is either mechanical [1]-[16], [18], [19], [22], [23], thermal [17], optical [20], [21], or electromagnetic [24]. Those loadings above are all independent of the deflection/ displacement of the structure; snap-through occurs but pull-in does not in those structures (modeling). In contrast, electrostatic and vdw forces are dependent on the structure deflection/ displacement. As the MEMS structure deflects more, the gap between the structures shrinks and those attractive (electrostatic and vdw) forces increase nonlinearly until pull-in. In those pullin studies [25]-[31], the initial configurations of the structures are explicitly or implicitly assumed flat, and snap-through, thus, cannot occur. However, for the structures with a flat initial configuration, the constant residual stress or thermal loading sometimes can be so large that the microstructures enter the postbuckling region with a curvy equilibrium configuration [32],[33]. Furthermore, because the residual stress gradients, which vary through the thickness, generate a bending moment, the microstructure instantly curves up or down after releasing [34]. Therefore, under an electrostatic loading, the structure with a curved (initial) equilibrium configuration might experience both the snap-through and pull-in instabilities. At the same time, many MEMS devices are designed with the initial curved/V-shaped configurations in order to utilize the snapthrough instability as an actuation mechanism [16]-[24]. The pull-in instability is not expected for those MEMS devices
[16]-[24] in modeling sense because of their loading types analyzed above. However, in reality, electrostatic voltage could be left over during the fabrication to become a pronounced factor affecting the behavior of structures [26], and pull-in may happen for those curved/V-shaped MEMS devices [16]-[24] if the left-over electrostatic voltage is large enough. So far, the study of combining the two instabilities, to the authors' best knowledge, has not been presented.

In this paper, we first examine these two instabilities for a one-degree-of-freedom (one-DOF) system. As the analysis shows that both snap-through and pull-in instabilities are a saddle node type of bifurcation (or fold catastrophe) [1], [2], their differentiation lies in the physical aspect. For snap-through, the structure does not collide with the electrode substrate, whereas for pull-in, the collision happens. In addition, for the structure with a certain initial curved configuration, snap-through does not necessarily happen as the external load increases [13], [14], [22], [35]; but pull-in happens for sure when the (electrostatic) attractive load reaches the critical value. The model for a beam with an initial curved configuration under an electrostatic loading and its numerical computation are presented. The computational results are also compared with the experiments. Two types of responses are found: the structures either collide with the substrate without snap-through or snap-though first and then collide. As the pull-in instability results in the collision and failure of the MEMS system, there is a maximum displacement for the structure to reach without pull-in. Because the larger maximum structure displacement can provide better sensitivity and tuning range, a large range of structure deflection can be extremely useful for a wide variety of tuning applications [27]. The axial loading is shown to be a parameter that can change the structure maximum displacement of a flat beam [29], [31]. Here, the initial curved configuration is also demonstrated to be an effective way of enlarging the maximum displacement.

\section{SNAP-Through AND PUll-In Instabilities OF A ONE-DOF SYSTEM}

Fig. 1(a) shows two trusses with length $L$ tied with a spring $K_{1}$. The trusses are rigid and pinned at an apex. $\alpha$ is the initial angle, and $Q_{1}$ is the angle with the presence of load $P$. The equilibrium equation for this one-DOF tied arch is given as follows [1]:

$$
F=\frac{P}{4 K_{1} L}=\left(\cos Q_{1}-\cos \alpha\right) \tan Q_{1} .
$$

Equation (1) gives the equilibrium path of the system, as shown in Fig. 2. Fig. 2 plots the $F-\left(\alpha-Q_{1}\right)$ curve, where $F$ is treated as a control parameter. Physically, $\alpha-Q_{1}$ is the system rotation angle, and the initial angle $\alpha$ is set as $\pi / 36$. For the loading process, $F$ starts with zero and increases. The corresponding starting point in Fig. 2 is $B$ when $F=0$ and $\alpha-Q_{1}=0$. The system follows the $B C$ curve until point $C$ during the loading process. At $C$, the slope is infinite, and two curves of $B C$ and $E C$ meet. In Fig. 2, the solid line indicates the stable equilibrium and the dashed line for the unstable one. $C$ is the point where the stable and unstable equilibria meet. At $C$, if $F$ is increased further (infinitesimally), snap-through 
happens. Equilibrium jumps from $C$ to $D$, which results in the discontinuous displacement change, and the whole structure turns upside down, as shown in Fig. 2. When $F$ is unloaded, the equilibrium will follow the $D E$ curve until point $E$. At $E$, the slope is again infinite, and any infinitesimal decrease of $F$ will again cause snap-through and the equilibrium jumps from $E$ to $A$. It needs to be emphasized here that the snapthrough model above is a particular one for a one-DOF system. $2 L \cos \alpha$ is the spring original length, and $2 L \cos Q_{1}$ is the length with a load $P$. Before the trusses snap-through, $\alpha>0$, $Q_{1}>0$, and $\alpha \geq Q_{1}$, so $2 L \cos \alpha \leq 2 L \cos Q_{1}$, which physically means that, after lateral loading, the spring elongates, and the spring force becomes tensile. The elongation is guaranteed by the rollers, as indicated in Fig. 1(a). However, for the beam structure of a continuous system, as analyzed later, the ends are not movable or very stiff [35]; and the various loadings, including the compressive one, can thus be exerted at the ends. Johnson et al. show that there are three types of the arched beam response: no instability, snap-through instability at a limit point, and snap-through instability at a bifurcation point [35]. For an arched beam with the two ends fixed, the beam span is actually shortened during its deflection before the snap-through under a lateral load. Because of this, and also depending on the initial thrust at the ends, the axial force inside the beam can be compressive. If the compressive axial load is so large, the structure can lose stability due to buckling (pitchfork bifurcation). When the buckling happens, the beam loses resistance to the lateral load, and snap-through occurs, which is the type of snapthrough at a bifurcation point [35]. Equation (1) is incapable of predicting this type of response, as analyzed above that the oneDOF model always predicates the elongation of the spring.

Fig. 1(b) shows a plate with a spring $K_{2}$ under an electrostatic loading. The governing equation, which describes the balance of the elastic spring force and electrostatic force of this one-DOF system, is given as follows [31]:

$$
K_{2} w=\frac{C}{(d-w)^{2}}
$$

where $w$ is the plate displacement, $d$ is the gap distance between the plate and the electrode before actuation, $K_{2}$ is the spring stiffness, and $C$ is a constant given as $C=\epsilon A V^{2} / 2$ [27], [29], [31]. ( $\epsilon$ : dielectric constant of air; $A$ : plate area; and $V$ : voltage.) By simple manipulation, (2) is nondimensionalized and rewritten as follows:

$$
y^{3}-2 y^{2}+y-C_{o}=0 .
$$

Here, $y$ is the dimensionless variable defined as $y=w / d$ and $C_{o}=C /\left(K_{2} d^{3}\right)$. Equation (3) is a cubic equation and can be analytically solved by the Cardan solution [36]. In a cubic equation, $Q$ is the parameter that indicates the solution scenarios of the three roots. $Q$ is given as

$$
Q=\left(\frac{p}{3}\right)^{3}+\left(\frac{q}{2}\right)^{2} .
$$

Here, $p=-\left((-2)^{2} / 3\right)+1=-(1 / 3)$, and $q=2(-2 / 3)^{3}-$ $(1 / 3)(-2)-C_{o}=(2 / 27)-C_{o}$.

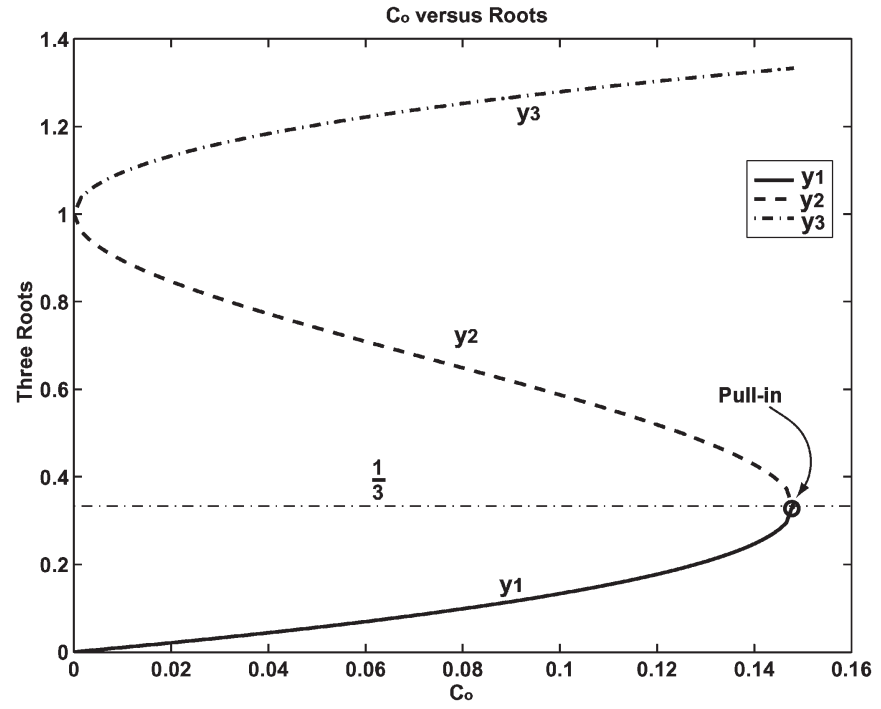

Fig. 3. Pull-in instability of a plate under an electrostatic load and its three roots/equilibria with the change of $C_{o}$.

When $Q<0$, there are three real roots. When $Q=0$, there are three real roots, where at least two roots are the same. When $Q>0$, there are 1 real roots, 2 complex conjugate roots. The three roots of (3) are given in Fig. 3, as $C_{o}$ changes from 0 to $4 / 27$. The three real roots exist until $C_{o}=4 / 27$. At $C_{o}=4 / 27, Q=0$. After $C_{o}=4 / 27$, only $y_{3}$ exists in the real domain. $y_{3}$ can be physically excluded as a solution because $y_{3} \geq 1$, which means the penetration of the plate through the electrode. $y_{2}$ can be excluded as a solution because it is an unstable solution. Therefore, only $y_{1}$ is the stable and physically reasonable solution for (3). In Fig. 3, it is shown that, at $C_{o}=4 / 27, y_{1}=y_{2}=1 / 3$, and this $1 / 3$ is the maximum dimensionless displacement of the one-DOF plate model before the pull-in instability [27], [31]. When $C_{o}>4 / 27, y_{1}$ and $y_{2}$ cease existing in the real domain, and only $y_{3}$ is left in the real domain. By further increasing $C_{o}$, the solution will jump from $y_{1}$ to $y_{3}$ in the real domain, which indicates the pull-in instability and that the structure collides with the electrode.

Now let us examine and compare these two instabilities of snap-through and pull-in. It first appears that the two equations of (1) and (3) govern two types of different instability phenomenon. However, the readers should be aware that there are other modelings for snap-through and pull-in. The two oneDOF examples presented in this paper are the particular cases. For example, the snap-through model of two elastic trusses by Nachbar and Huang [8] gives the cubic equation that is similar to the pull-in instability governing (3). As shown in both Figs. 2 and 3, for both snap-through and pull-in, the slopes of the curves become infinite at the critical points, and their curves share the same pattern: that three solutions/equilibria coexist in a certain loading range. Their equilibria both jump at the critical fixed loads. In the mechanics point of view, these two instabilities are the same type of saddle node bifurcation (fold catastrophe) [1], [2]. Physically, when pull-in happens, the equilibrium $y_{1}$ tries to jump to $y_{3}$. However, as $y_{3}>1$, the plate must hit the substrate first before it can reach the stable equilibrium of $y_{3}$. Once the plate hits and adheres to the 
(a)

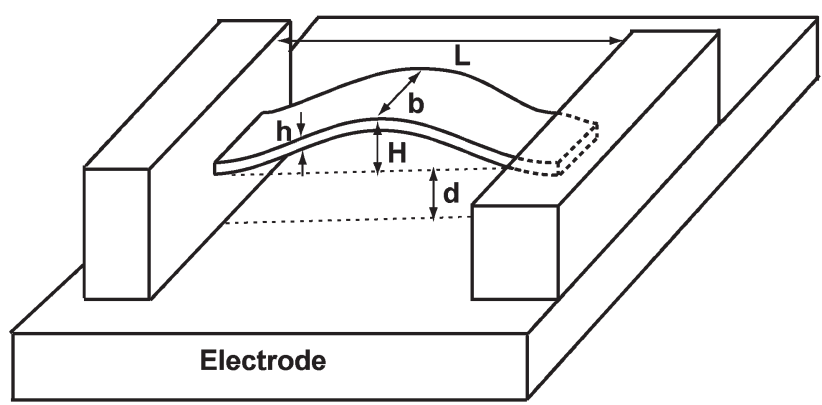

(b)

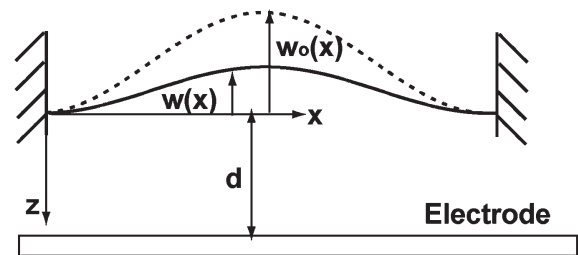

Fig. 4. (a) Dimensions of the system. (b) Coordinate system.

substrate, the governing equation of (3) is no longer valid, and other types of equation should be introduced [37]. In contrast to the pull-in instability, the snap-through instability can be repeated in loading-unloading cycles. In the following study of the beam with the initial curved configuration under an electrostatic loading, snap-through is defined as the one that the beam flips but does not hit the substrate, and pull-in is the one that hits the substrate.

\section{ARCh-Shaped BeAm Under AN ELECTROSTATIC LOADING}

In this section, the continuous system of an arch-shaped beam under an electrostatic loading is studied. Fig. 4(a) shows the schematic diagram and dimensions of the system. $L, b$, and $h$ are the span, width, and thickness of the beam, respectively. The beam is clamped at the two ends. $d$ is the gap distance between the beam ends and electrode, and $H$ is the midspan arch rise from the beam ends. The coordinate system is indicated in Fig. 4(b). The governing equation for an arch-shaped beam under a distributed load is given as follows [6], [7], [9], [11], [12]:

$$
E^{*} I \frac{d^{4}\left(w-w_{o}\right)}{d x^{4}}+P \frac{d^{2} w}{d x^{2}}+P_{o} \frac{d^{2} w_{o}}{d x^{2}}+q(x)=0 .
$$

The governing equations of those studies [6], [7], [9], [11], [12] are for dynamics of an arch-shaped beam. Here, only the static response is studied, and therefore, (5) truncates those time-related terms. $E^{*}$ is the effective Young's modulus of the beam. For the narrow beam, $E^{*}=E$ is the Young's modulus of the beam. For the wide beam bending in a cylindrical surface, $E^{*}=E /\left(1-\nu^{2}\right)$, and $\nu$ is Poisson's ratio of the beam [29]. $I$ is the moment of inertia of the cross section, and $I=b h^{3} / 12$ for a rectangular cross section. $w$ and $w_{o}$ are the deformed and initial coordinates of the beam centerline measured from the $x$-axis, as shown in Fig. 4(b). $P$ is defined as $P=-P_{o}+$ $\left(E^{*} A / 2 L\right) \int_{0}^{L}\left[\left(d^{2} w_{o} / d x^{2}\right)^{2}-\left(d^{2} w / d x^{2}\right)^{2}\right] d x . A=b h$ is the cross-sectional area, and $(1 / 2) \int_{0}^{L}\left[\left(d^{2} w_{o} / d x^{2}\right)^{2}-\left(d^{2} w /\right.\right.$ $\left.\left.d x^{2}\right)^{2}\right] d x$ is the (nonlinear) axial displacement due to the beam midplane stretching. $P_{o}$ is the initial thrust, and $P_{o}$ here has the opposite sign as that defined by Hsu [9] in order to comply with the rule that a positive $P_{o}$ indicates tension and a negative $P_{o}$ indicates compression [29], [31]. $q(x)$ is the distributed transverse load. Here, $q(x)$ is the electrostatic loading, and $q=-\left(\epsilon b V^{2} / 2(d-w)^{2}\right) . \epsilon$ is the dielectric constant of air, and $V$ is the applied voltage. With the substitutions of $P$ and $q$, (5) is rewritten as follows:

$$
\begin{aligned}
& E^{*} I \frac{d^{4}\left(w-w_{o}\right)}{d x^{4}} \\
& +\left\{-P_{o}+\frac{E^{*} A}{2 L} \int_{0}^{L}\left[\left(\frac{d^{2} w_{o}}{d x^{2}}\right)^{2}-\left(\frac{d^{2} w}{d x^{2}}\right)^{2}\right] d x\right\} \frac{d^{2} w}{d x^{2}} \\
& +P_{o} \frac{d^{2} w_{o}}{d x^{2}}-\frac{\epsilon b V^{2}}{2(d-w)^{2}}=0
\end{aligned}
$$

When $w_{o}(x)=0$, (6) recovers the equation of equilibrium that was derived by Abdel-Rahman et al. [29] for a flat beam under an electrostatic loading. The above governing equation is for a shallow arch model that takes into account the nonlinearity effect of midplane stretching only (the $(1 / 2 L) \int_{0}^{L}\left[\left(d^{2} w_{o} / d x^{2}\right)^{2}-\left(d^{2} w / d x^{2}\right)^{2}\right]$ term). For a deep arch model, the elastica model is needed for a much more complex stress-strain relation in the structure [13], [14]. It is also worth mentioning that (6) is capable of studying the buckled microstructures [32], [33]. When the electrostatic force term of $\epsilon b V^{2} / 2(d-w)^{2}$ is set to zero, (6) recovers the governing equation that describes the buckling and postbuckling of the microstructures given by Fang and Wickert [32]. If a comparison is made for the continuous system of a beam with the one-DOF system, there are two springs in (6): the axial spring with the effective spring constant $k_{x}=E^{*} A / L$ and the transverse spring with the effective spring constant $k_{z} \propto E^{*} I / L^{3}$ [38]. $k_{x}$ is the spring corresponding to $K_{1}$ in Fig. 1(a), and $k_{z}$ is the one corresponding to $K_{2}$ in Fig. 1(b).

To nondimensionalize (6), the same nondimensionalization scheme as that of Abdel-Rahman et al. [29] is used, and the following quantities are introduced:

$$
W_{o}=\frac{w_{o}}{d} \quad W=\frac{w}{d} \quad \xi=\frac{x}{L} .
$$

The dimensionless governing equation is given as follows:

$$
\begin{aligned}
& \frac{d^{4}\left(W-W_{o}\right)}{d \xi^{4}} \\
& +\left\{-\alpha_{1}+\alpha_{2} \int_{0}^{1}\left[\left(\frac{d^{2} W_{o}}{d \xi^{2}}\right)^{2}-\left(\frac{d^{2} W}{d \xi^{2}}\right)^{2}\right] d \xi\right\} \frac{d^{2} W}{d \xi^{2}} \\
& \quad+\alpha_{1} \frac{d^{2} W_{o}}{d \xi^{2}}-\frac{\alpha_{3} V^{2}}{(1-W)^{2}}=0
\end{aligned}
$$

where the $\alpha_{i}$ 's ( $i=1$ to 3 ) are defined as follows:

$$
\alpha_{1}=\frac{P_{o} L^{2}}{E^{*} I} \quad \alpha_{2}=6\left(\frac{d}{h}\right)^{2} \quad \alpha_{3}=\frac{6 \epsilon L^{4}}{E^{*} h^{3} d^{3}} .
$$


For the convenience of computation, (8) is written in the following form as Humphreys did [6]:

$$
\begin{aligned}
& \frac{d^{4}(\Delta W)}{d \xi^{4}}+\left\{-\alpha_{1}+\alpha_{2} \int_{0}^{1}\right. {\left[\left(\frac{d^{2} W_{o}}{d \xi^{2}}\right)^{2}\right.} \\
&\left.\left.-\left(\frac{d^{2} W_{o}}{d \xi^{2}}+\frac{d^{2} \Delta W}{d \xi^{2}}\right)^{2}\right] d \xi\right\} \\
& \times\left(\frac{d^{2} W_{o}}{d \xi^{2}}+\frac{d^{2} \Delta W}{d \xi^{2}}\right)+\alpha_{1} \frac{d^{2} W_{o}}{d \xi^{2}}-\frac{\alpha_{3} V^{2}}{\left(1-W_{o}-\Delta W\right)^{2}}=0 .
\end{aligned}
$$

Here, $\Delta W(\xi)=W(\xi)-W_{o}(\xi)$ is the dimensionless displacement of the beam. In order to compute (10) numerically, the Galerkin method [29]-[31] is used here. $\Delta W$ is first discretized as follows:

$$
\Delta W=\sum_{i=1}^{N} a_{i} \phi_{i}(\xi) .
$$

$N$ is the mode number. $a_{i}$ is the constant modal amplitude to be determined. $\phi_{i}$ is the $i$ th mode shape of the clamped-clamped beam. $\phi_{i}=1 / 2\left[\delta_{i} e^{\beta_{i} \xi}+\gamma_{i} e^{-\beta_{i} \xi}\right]-$ $\cos \left(\beta_{i} \xi\right)+\alpha_{i} \sin \left(\beta_{i} \xi\right) ; \delta_{i}, \gamma_{i}, \beta_{i}$, and $\alpha_{i}$ are the constants given by Chang and Craig [39] for each $\phi_{i}$. By substituting (11) into (10), multiplying by $\phi_{i}(i=1$ to $N)$, and integrating from 0 to 1 , the set of nonlinear equations, shown by (12) at the bottom of the page, is obtained. Here, ()$^{\prime}=d / d \xi$. There are a total of $N$ nonlinear equations for $N$ unknown $a_{i}$ 's. With the discretization of the Galerkin method, the above equations are a polynomial equation set of $a_{i}$ 's. The Newton-Rahpson method is applied to find the $a_{i}$ 's, and the related computer codes are taken from [40].

\section{FABRICATION AND MEASUREMENT}

In this paper, the Silicon Glass Anodic-bonding and Deep Etching Release (SGADER) process [41], which was developed at Peking University, is used to fabricate the testing device. The basic processing steps are shown in Fig. 5. The process of fabricating mechanical structures combines wafer bonding and deep reactive ion etching (DRIE) technologies. The starting materials are 4-in medium-doped silicon wafers and Pyrex 7740

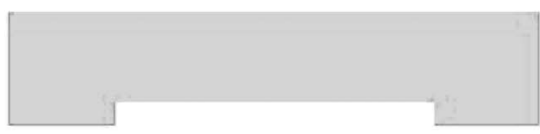

(a)

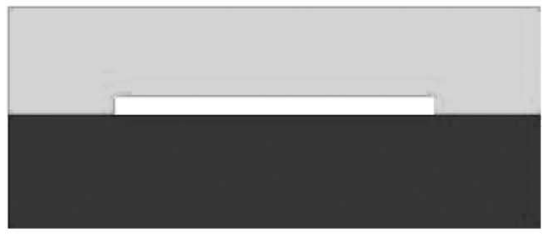

(b)

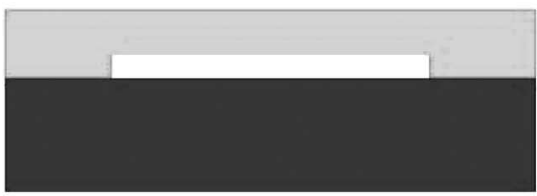

(c)

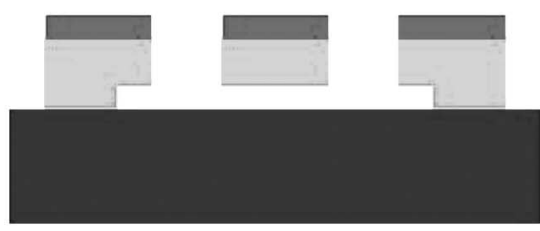

(d)

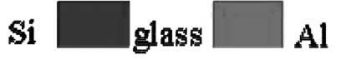

Fig. 5. Two-mask SGADER process flow. (a) Shallow trench made by $\mathrm{KOH}$. (b) Silicon/Glass anodic bonding. (c) Silicon wafer thinned by KOH. (d) Structure releasing by deep RIE.

glass wafers. First, a shallow trench about $10 \mu \mathrm{m}$ is etched by potassium hydroxide $(\mathrm{KOH})$ to shape the anchors [Fig. 5(a)], which are used to sustain the movable beams and the electrodes. After the silicon dioxide is stripped by buffered HF (BHF), the silicon and glass wafers are then anodically bonded together [Fig. 5(b)] under the conditions of $380{ }^{\circ} \mathrm{C}$ and $-1500 \mathrm{~V}$. The silicon wafers are thinned to about $50 \mu \mathrm{m}( \pm 5 \mu \mathrm{m})$ by $\mathrm{KOH}$ etching [Fig. 5(c)]. Next, the beam and electrode structures are released through DRIE, and a sputtered aluminum layer is used as a mask [Fig. 5(d)]. After this step, the standard SGADER process is finished, and the device is fabricated. Fig. 6, which shows a 3-D view of the structure in test, together with Fig. 5,

$$
\left\{\begin{array}{c}
\int_{0}^{1} \phi_{1}\left\{\sum_{i=1}^{N} a_{i} \phi_{i}^{\prime \prime \prime \prime}+\left\{-\alpha_{1}+\alpha_{2} \int_{0}^{1}\left[W_{o}^{\prime \prime 2}-\left(W_{o}^{\prime \prime}+\sum_{i=1}^{N} a_{i} \phi_{i}^{\prime \prime}\right)^{2}\right] d \xi\right\}\left(W_{o}^{\prime \prime}+\sum_{i=1}^{N} a_{i} \phi_{i}^{\prime \prime}\right)+\alpha_{1} W_{o}^{\prime \prime}-\frac{\alpha_{3} V^{2}}{\left(1-W_{o}-\sum_{i=1}^{N} a_{i} \phi_{i}\right)^{2}}\right\} d \xi=0 \\
\int_{0}^{1} \phi_{2}\left\{\sum_{i=1}^{N} a_{i} \phi_{i}^{\prime \prime \prime \prime}+\left\{-\alpha_{1}+\alpha_{2} \int_{0}^{1}\left[W_{o}^{\prime \prime 2}-\left(W_{o}^{\prime \prime}+\sum_{i=1}^{N} a_{i} \phi_{i}^{\prime \prime}\right)^{2}\right] d \xi\right\}\left(W_{o}^{\prime \prime}+\sum_{i=1}^{N} a_{i} \phi_{i}^{\prime \prime}\right)+\alpha_{1} W_{o}^{\prime \prime}-\frac{\alpha_{3} V^{2}}{\left(1-W_{o}-\sum_{i=1}^{N} a_{i} \phi_{i}\right)^{2}}\right\} d \xi=0 \\
\vdots \\
\int_{0}^{1} \phi_{N}\left\{\sum_{i=1}^{N} a_{i} \phi_{i}^{\prime \prime \prime}+\left\{-\alpha_{1}+\alpha_{2} \int_{0}^{1}\left[W_{o}^{\prime \prime 2}-\left(W_{o}^{\prime \prime}+\sum_{i=1}^{N} a_{i} \phi_{i}^{\prime \prime}\right)^{2}\right] d \xi\right\}\left(W_{o}^{\prime \prime}+\sum_{i=1}^{N} a_{i} \phi_{i}^{\prime \prime}\right)+\alpha_{1} W_{o}^{\prime \prime}-\frac{\alpha_{3} V^{2}}{\left(1-W_{o}-\sum_{i=1}^{N} a_{i} \phi_{i}\right)^{2}}\right\} d \xi=0
\end{array}\right.
$$




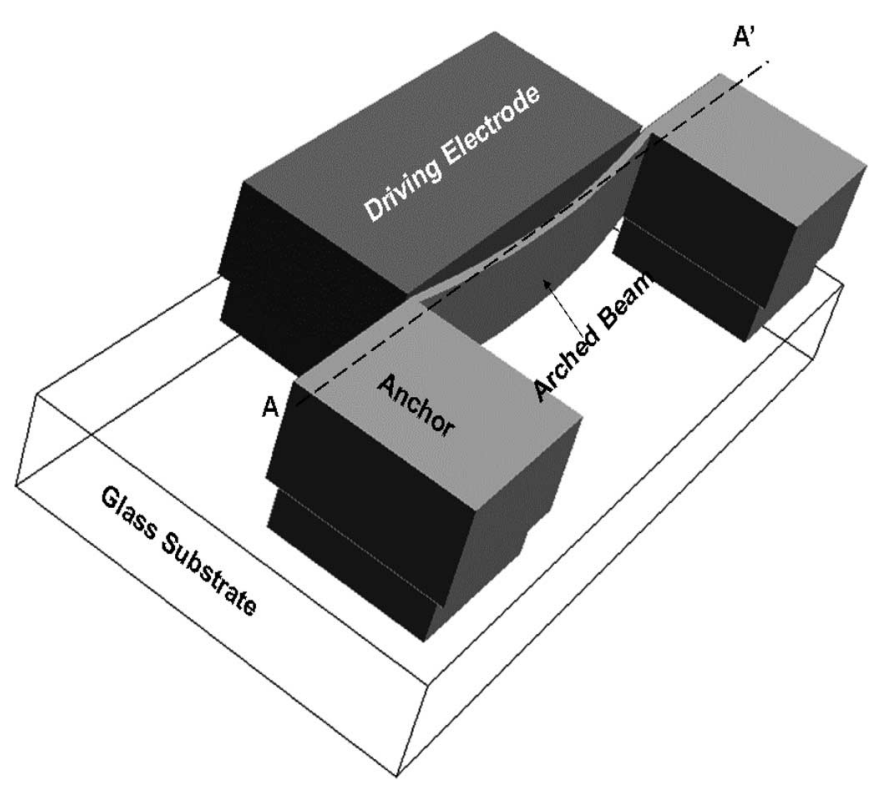

Fig. 6. Three-dimensional view of the arch-shaped microbeam in the study.

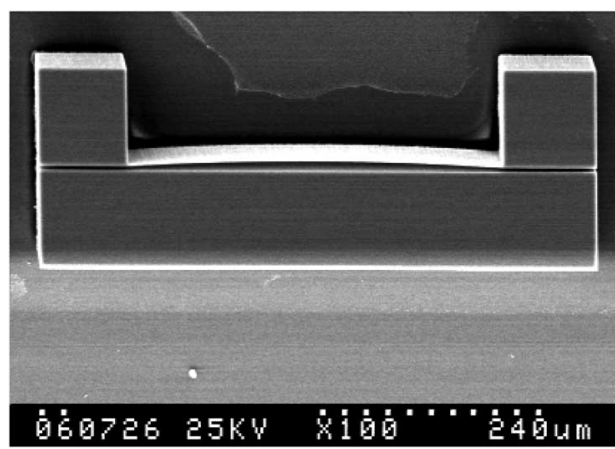

Fig. 7. SEM photograph of the arch-shaped microbeam in the study.

facilitates the reader to see how the arch-shaped beam geometry is formed. Fig. 5 is a cross-sectional view along the $A-A^{\prime}$ direction in Fig. 6. The arch-shaped beam with an out-of-plane arch configuration is hard to be controlled using the planar microfabrication. Usually, the arch can be formed by utilizing the mechanisms of the stress mismatch inside different layers for a multilayer structure or the stress gradients varying through the thickness of a homogeneous structure. However, in practice, it is very difficult to control the beam parameters (for example, the arch rise $H$ and arch shape) with high precision when utilizing the above mechanisms. Here, a bulk micromaching technology is used here for the fabrication, which not only fabricates the beam with a high precision of beam dimensions by lithography, but also eliminates the residual stress by a wellcontrolled process [42]. Fig. 7 shows the scanning electronic microscope (SEM) photo of the fabricated arch-shaped beam in test.

The relative displacement of the arch-shaped beam with varying voltages is measured by a MEMS motion analyzer apparatus at the State Key Laboratory of Precision Measuring Technology and Instruments, Tianjin University. Fig. 8 shows the functional diagram of the MEMS measurement system based on computer microvision and phase-shifting interferometry. In the system, a video camera and a frame grabber are utilized to record the MEMS device motion/deflection in real time. An integrated computer control and data acquisition unit, which consists of a variety of standard internal plug-in cards and external general-purpose interface bus instruments, is used to supply power, generate signals, control voltages, and measure the MEMS motion/deflection automatically. A micromanipulator system, including a manual $\mathrm{X}-\mathrm{Y}-\mathrm{Z}$ micrometer position stage, is used for positioning the devices in test. The hardware platform includes an optical microscope with general objectives and Mirau interferometer objectives, a stroboscopic laser source, and a lead zirconate titanate phase shifter for imaging. A software analysis package processes the captured images and signals, and generates testing reports. The in-plane dimension and motion measurement of MEMS devices are done by image matching of computer microvision; the out-ofplane dimension and motion measurement MEMS devices are achieved by a two-beam microscopic interferometry with a fivestep phase shifting.

\section{Results And Discussion}

The beam is made of silicon, with $E=160 \mathrm{GPa}$ and $\nu=0.27$. For all the beams computed and tested here, $b$ is fixed as $b=50 \mu \mathrm{m}$. $E^{*}$ is defined as follows: $E^{*}=E /(1-$ $\left.\nu^{2}\right)$. The dielectric constant of air is $\epsilon=8.854 \times 10^{-12} \mathrm{~F} / \mathrm{m}$. The arch is an arc of a circle, and for a shallow arch, we use a parabolic curve to approximate $w_{o}$ for simplicity; therefore, $w_{o}(x)=4 H\left(x^{2}-L x\right) / L^{2}$. The initial thrust $P_{o}=$ $-\left(E^{*} A / 2 L\right) \int_{0}^{L}\left(d w_{o} / d x\right)^{2} d x=-\left(8 E^{*} b h H^{2} / 3 L^{2}\right)$. As the computation accuracy is sensitive to the mode number $N$ [7], [30], [31], we first do the convergence study for (12). Fig. 9 plots the dimensionless displacement of the beam center $\Delta W(0.5)$ as a function of the applied voltage $V$ for different mode numbers $N$. The related dimensions are $L=500 \mu \mathrm{m}$, $H=2.9 \mu \mathrm{m}, d=2.9 \mu \mathrm{m}$, and $h=2.6 \mu \mathrm{m}$. The voltage increases until pull-in. Clearly, there is a significant difference between the result of $N=1$ and those of $N=3$ and $N=5$. The physical reason is that, with the increase in the applied voltage, the participation of the (symmetric) third mode $\phi_{3}$ becomes more and more significant. Furthermore, for $N=1$, there is a numerical fluctuation around the critical pull-in point. As the system approaches pull-in, the slope of the curve becomes larger and larger, and the computed $\Delta W(0.5)$ becomes more and more sensitive to the step size of the applied voltage. Any tiny change of voltage can cause the large change of $\Delta W(0.5)$. This is the why the results of $N=3$ and $N=5$ agree with each other at all points, except the pull-in point. Both $N=3$ and $N=5$ predict the same pull-in voltage of $V=87 \mathrm{~V}$, but the pull-in $\Delta W(0.5)=0.168$ for $N=3$, and $\Delta W(0.5)=0.201$ for $N=5$. Although there is an accuracy control mechanism in the algorithm of the Newton-Rahpson method used in our numerical computation [40], the fact that the slope approaches infinity can still cause the significant computation error of the maximum pull-in displacement. The similar scenario also happens in the pull-in computations of two nanotubes under the influence of the vdw force [30] and different flat beams under an electrostatic loading [31]. The odd modes of the clamped-clamped beam $\phi_{i}$ 's $(i=1,3,5,7, \ldots)$ are symmetric, 


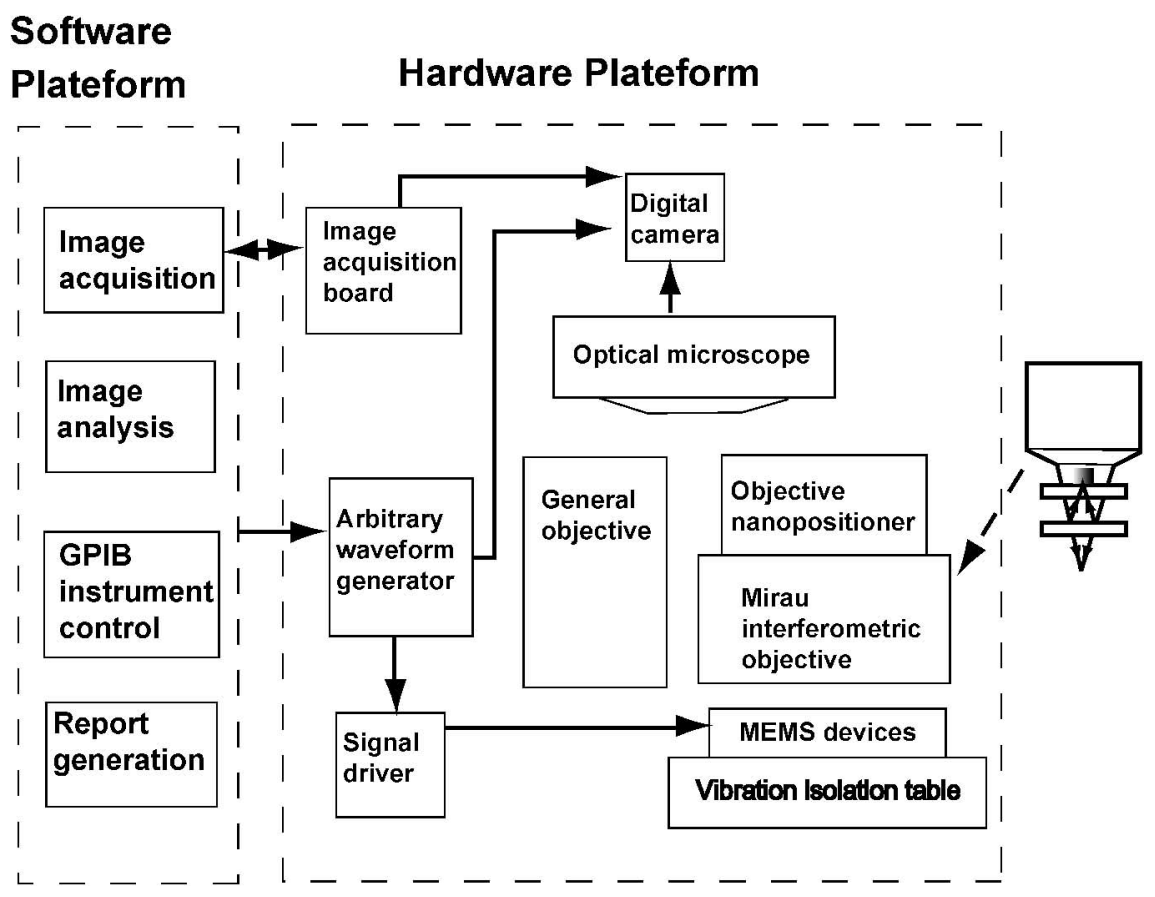

Fig. 8. Functional diagram of the software and hardware measuring systems.

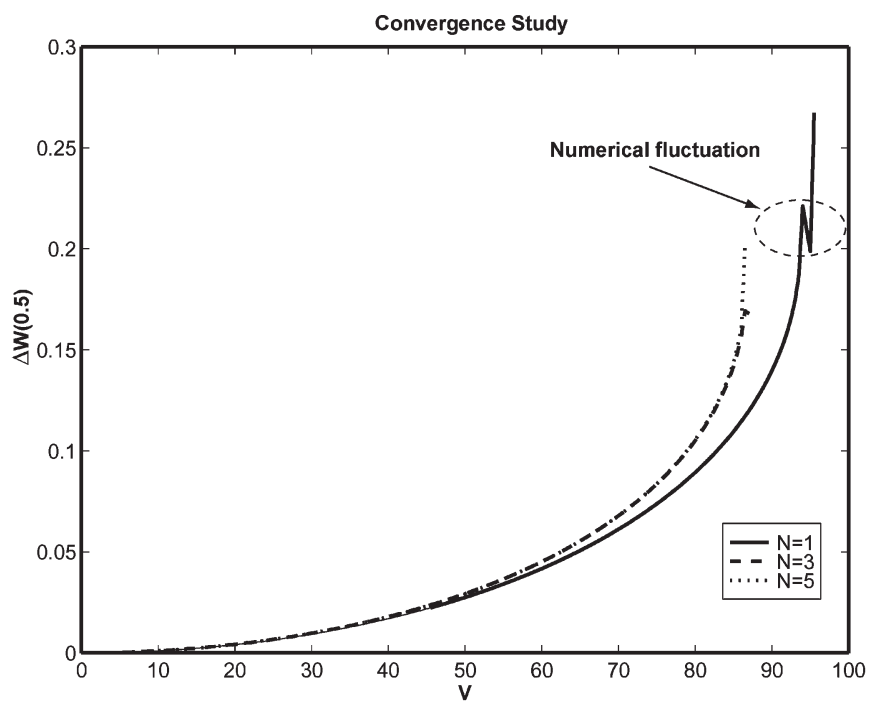

Fig. 9. Convergence study of the Galerkin method for different mode numbers $N$.

whereas the even modes of $\phi_{i}$ 's $(i=2,4,6,8, \ldots)$ are antisymmetric. For the flat symmetric structure under a symmetric loading, using only odd modes to discretize $\Delta W$ in (11) is proved to be an effective method to reduce both numerical fluctuation and computation effort [31]. However, caution should be taken for the case of the arch-shaped beam with the symmetric configuration and symmetric loading. As demonstrated by both the static and dynamic studies of a symmetric arch-shaped beam under a symmetric loading, the asymmetric deflection appears in both the static experiment [13] and model [14], and the antisymmetric mode significantly participates in the motion, causing a $70 \%$ difference of critical snap-through load in a dynamic model [7]. This phenomenon that the asymmetric deflection or motion is induced in a symmetric arch-shaped beam under a symmetric loading is somewhat counterintuitive. Johnson et al. [35] introduce a dimensionless thrust parameter and analytically show that when this parameter reaches a certain value, an antisymmetric deflection solution will become a part of total deflection solution, and the deflection becomes asymmetric. Although the contribution of antisymmetric modes in the arch deflection is found trivial in all our computations, we still keep both odd and even modes, and $N$ is taken as 5 . In our model and experiment, the arch is shallow, and the load is the distributed electrostatic force. In those two static studies above [13], [14], the arch is deep, and the load is a concentrated mechanical force; the symmetric deflection also appears, and the initial arch configurations play the vital role of determining whether the symmetric or asymmetric deflection occurs. The participation of antisymmetric mode in Lock's study of a shallow arch [7] is purely due to the dynamic effect, as reflected from the governing equation of the Mathieu equation.

Fig. 10 plots the responses of two arch-shaped beams under an electrostatic loading. Case 1 has the following configurations: $L=500 \mu \mathrm{m} ; H=2.9 \mu \mathrm{m} ; d=6.4 \mu \mathrm{m}$; and $h=$ $2.6 \mu \mathrm{m}(H / h=1.12)$. Its experimental data are marked with the cross $(+)$ sign, and the solid line nearby is the numerical results. Case 2 has the following configurations: $L=500 \mu \mathrm{m}$; $H=2.6 \mu \mathrm{m} ; d=8.3 \mu \mathrm{m}$; and $h=2.6 \mu \mathrm{m}(H / h=1)$, and its experimental data are marked with a circle (o). With the increase in voltage, snap-through happens at point $A$ for case 1 , and the beam then jumps to point $B$. With the voltage increasing further, pull-in happens at point $C$. For case 2, snap-through happens at point $D$, the beam jumps to $E$, and pull-in happens at point $F$. As shown in Fig. 10, the numerical predictions overestimate both the critical voltages of snap-through and pull-in for both cases, as compared with the experimental data. For case 1, the snap-through voltage is $112 \mathrm{~V}$ (numerical) and $104 \mathrm{~V}$ (experiment); the pull-in voltage is $150 \mathrm{~V}$ (numerical) 


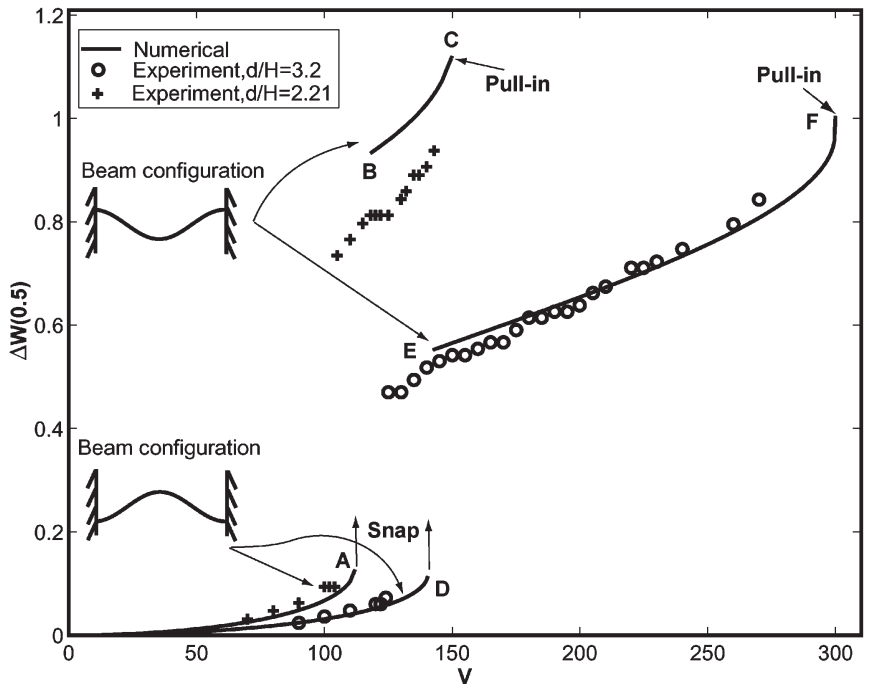

Fig. 10. Comparison of the model and experimental data of two arch-shaped beams. Snap-through first occurs and then pull-in happens with further increase in the voltage. The experimental data marked with + pertain to the beam with $L=500 \mu \mathrm{m}, H=2.9 \mu \mathrm{m}, d=6.4 \mu \mathrm{m}$, and $h=2.6 \mu \mathrm{m}(H / h=1.12)$. The other data marked with $\circ$ pertain to the beam with $L=500 \mu \mathrm{m}, H=$ $2.6 \mu \mathrm{m}, d=8.3 \mu \mathrm{m}$, and $h=2.6 \mu \mathrm{m}(H / h=1)$.

and $143 \mathrm{~V}$ (experiment). For case 2, the snap-through voltage is $140.5 \mathrm{~V}$ (numerical) and $124 \mathrm{~V}$ (experiment); the pull-in voltage is $300 \mathrm{~V}$ (numerical) and $270 \mathrm{~V}$ (experiment). There is around $10 \%$ error for the snap-through voltages for both cases. Here, some possible mechanisms that can cause this $10 \%$ difference between the numerical predication and experiment are discussed. First, when silicon is exposed, an oxide film forms at its surface, and the beam becomes inhomogeneous [16]. This inhomogeneity will cause the change of effective $E^{*} I$ and $E^{*} A$, which is not taken into accounte in (6). When the oxide film is very thin, the inhomogeneity influence is trivial. However, the compressive residual stress induced by the oxide film [16] could be significant, and (6) does not take into account this residual stress effect either. Second, the beam ends experience larger stress than the other parts of the beam, and plastic deformation could thus happen around the ends. The plastic deformation induces additional axial displacement, or during the deflection, the beam axial force pushes the end supports away, which also causes additional axial displacement. This, in essence, will change the effective axial spring constant of $k_{x}=E^{*} A / L$. Hsu introduces a parameter $\alpha$, which is called the equivalent spring constant of the elastic supports, and these two springs (i.e., $k_{x}$ and $\alpha$ ) are in serial configuration [9]. The effective axial constant becomes $k_{\text {eff }}=\left(E^{*} A \alpha / 2\left(E^{*} A+\right.\right.$ $\alpha L))\left(\left(1 / k_{\text {eff }}\right)=\left(1 / k_{x}\right)+(1 / \alpha)\right)$ and $k_{\text {eff }}<k_{x}$. Therefore, the effective stiffness $K_{1}$ becomes smaller, and as indicated in (1), smaller $K_{1}$ results in a smaller snap-through load $P$. Third, all the dynamic studies [6]-[12] demonstrate that the dynamic critical snap-through load is always smaller than the static one. Equation (6) is a static equation. During our experiment, the voltage is increased very slowly to avoid the dynamic effect. However, as analyzed above, when the voltage approaches the critical snap-through value, the curve slope becomes very large. A tiny load change can cause large deflection, and motion is thus easily induced. Therefore, the dynamic effect is brought

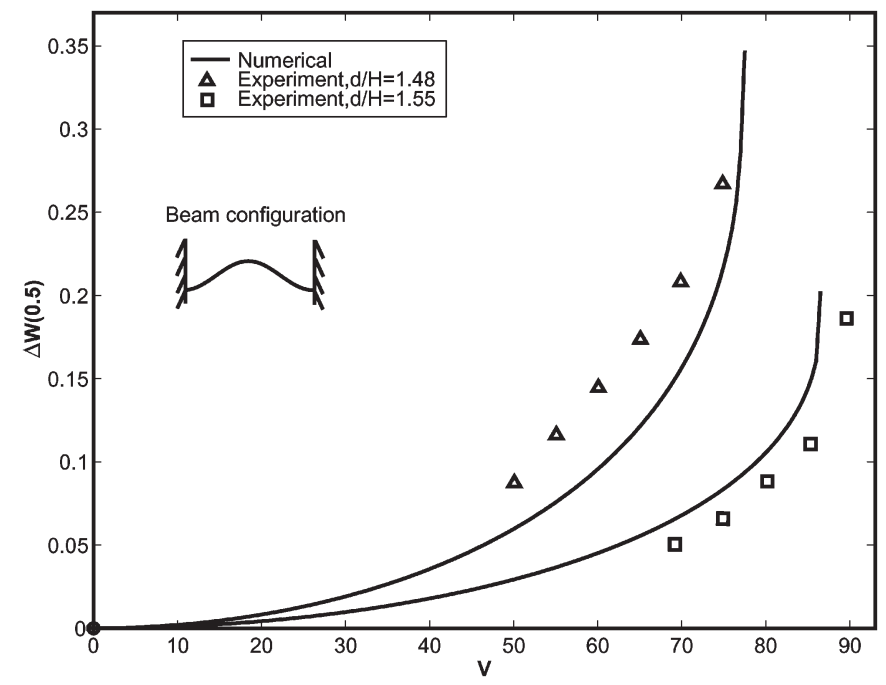

Fig. 11. Comparison of the model and experimental data of two arch-shaped beams. For these two beams, no snap-through happens. The beams directly hit the electrode substrate and adhere to it as pull-in happens. The experimental data marked with $\triangle$ pertain to the beam with $L=475 \mu \mathrm{m}, H=2.3 \mu \mathrm{m}$, $d=3.4 \mu \mathrm{m}$, and $h=2.6 \mu \mathrm{m}(H / h=0.885)$. The other data marked with $\square$ pertain to the beam with $L=500 \mu \mathrm{m}, H=2.9 \mu \mathrm{m}, d=4.5 \mu \mathrm{m}$, and $h=2.6 \mu \mathrm{m}(H / h=1.12)$.

in to cause the lower snap-through voltage value. Fourth, the axial load $P\left(P=-P_{o}+\left(E^{*} A / 2 L\right) \int_{0}^{L}\left[\left(d^{2} w_{o} / d x^{2}\right)^{2}-\right.\right.$ $\left.\left.\left(d^{2} w / d x^{2}\right)^{2}\right]\right)$ acting inside the arched beam also acts on the (clamped) ends, with an opposite direction, as Newton's third law applies. These forces acting on the ends that can be coupled with friction can induce unwanted moments at the ends [35]. Equation (6) and related boundary conditions are incapable of describing such a coupling effect. The first and second mechanisms in essence change the axial load $P$ in (6) and axial load also have significant influence on the pull-in voltage [29], [31]. All these mechanisms can also be the mechanisms that cause the difference between the numerical predication and experimental observation on the pull-in voltage. However, their influence degree on these two instabilities is not the same. As shown next in the arch-shaped beam with only the pull-in response, the pull-in voltage difference between the computation and experiment is much smaller.

Fig. 11 shows the other two types of beam response to the electrostatic loading. We name these two cases as cases 3 and 4 . The related dimensions are $L=475 \mu \mathrm{m}, H=2.3 \mu \mathrm{m}$, $d=3.4 \mu \mathrm{m}$, and $h=2.6 \mu \mathrm{m}$ (case $3, H / h=0.885$ ) and $L=$ $500 \mu \mathrm{m}, H=2.9 \mu \mathrm{m}, d=4.5 \mu \mathrm{m}$, and $h=2.6 \mu \mathrm{m}$ (case 4 , $H / h=1.12$ ). Unlike the two cases in Fig. 10, cases 3 and 4 do not experience snap-through. The beams do not flip before pull-in. Their instability pattern is exactly the same as the beam and plate with flat initial configurations [29], [31]. For case 3, the experimental data are marked with the $\triangle$ sign, and the solid line nearby is the numerical result. The experimental data of case 4 are marked with the $\square$ sign. Compared with the two cases in Fig. 10, the pull-in voltages in Fig. 11 that were predicted by the model agree fairly well with the experimental data. The pull-in voltage values in case 3 are $77.5 \mathrm{~V}$ (numerical) and $80 \mathrm{~V}$ (experiment); in case 4, they are $86.5 \mathrm{~V}$ (numerical) and $90 \mathrm{~V}$ (experiment). The dimensionless pull-in maximum 
displacements $\Delta W(0.5)$ for case 3 are 0.347 (numerical) and 0.265 (experiment). Those for case 4 are 0.203 (numerical) and 0.178 (experiment). The dimensionless pull-in maximum displacements (both numerical and experimental ones) of cases 3 and 4 are smaller than those of the clamped-clamped beam with flat initial configurations [29], [31]. However, those of cases 1 and 2 are significantly larger. Therefore, for those arch-shaped beams with both the snap-through and the pull-in instabilities, the arch configurations can enlarge the maximum displacement, which is very helpful for providing better sensitivity and tuning range [27].

It will be useful to tell when snap-through happens. The static studies of the arch-shaped beam with mechanical concentrated load show that the arch-shaped beam can deflect continuously without snap-through [13], [14], [22]. Qiu et al. give two criteria for snap-through to occur statically: 1 ) the ratio of the arch height to the beam thickness $(H / h)$ is large and 2) the (antisymmetric) second mode must be constrained [22]. Here, the arched beams in test are all shallow arches; $H / h=1.12$ of case $1, H / h=1$ of case $2, H / h=0.885$ of case 3 , and $H / h=1.12$ of case 4 . They all have small $H / h$ ratios, and some have snap-through and some do not. Clamping two or several curved beams together at their centers are demonstrated to be an effective method of constraining the second mode [16], [19], [22]. However, with the participation of the second mode in motion or deflection, the snap-through can still occur for shallow [7] or deep arch [13], [14]. Pippard and Patrício et al. give a clear phase diagram from which whether snap-through occurs or not can be told [13], [14]. Still, the phase diagram is obtained point by point by running through different arch configurations in the experiment or numerical computation. The loading in the cases of Pippard and Patrício et al. is a mechanical concentrated load. In our distributed electrostatic loading case, we face another difficulty of constructing such a diagram. In Section II, we point out that snap-through and pull-in are of the same type of instability. They both cause the infinity of the slope and displacement jump. We can only differentiate them by the physical criterion of whether the collision with the substrate occurs. In the response of cases 3 and 4 in Fig. 11, it could be the scenario that the axial spring $k_{x}$ first loses the capability of taking further the electrostatic loading (i.e., snap-through happens first), and then during the snap-through jump, the transverse spring $k_{z}$ also becomes incapable of withstanding the further loading (pullin happens). The final result is thus that the beam collides with the substrate, and this is the same scenario as when the pull-in instability happens first. We cannot differentiate these two scenarios from either our computation or the experimental observation. Therefore, in cases 3 and 4, we only observe pullin. Only when snap-through occurs first and the structure stays stable after jump as cases 1 and 2 in Fig. 10 can we observe that snap-through occurs. In the phase diagram of Pippard [13] and Patrício et al. [14], only two parameters (the beam span and the initial arch angle at the clamped ends) are used. In addition to the geometries of arch-shaped beam, other parameters like $P_{o}$ and $d$ in (6) are also important in determining snapthrough. It is just too many parameters to construct such a phase diagram.

\section{SUMMARY}

The snap-through and pull-in instabilities are analyzed and compared first for one-DOF system, and the study is then extended to the continuous system of the arch-shaped beam under an electrostatic loading. For the arch-shaped beam under an electrostatic loading, the snap-through may or may not occur, and the pull-in instability occurs for sure. The two types of response of arch-shaped beam under an electrostatic loading are demonstrated and analyzed. The numerical analysis overestimates the critical snap-through load, and the possible mechanisms are discussed. The arch configuration is responsible for the snap-through instability and contributes to the larger maximum displacement of the arch-shaped beam with the snapthrough response. Furthermore, by design, the beam initial arch shape $w_{o}(x)$ can be formed by other mechanisms such as the residual stress/gradient and thermal loading. The model presented here is a generalized one, which takes the structure initial imperfection into account. As long as the initial arch shape $w_{o}(x)$ is given, the model can be applied. The initial arch shape studied here is symmetric, and all structure deflections are found symmetric under a symmetric distributed electrostatic loading. The model presented in this paper is a static analysis. The effects of the asymmetry of initial configuration and dynamics are not included here and left for our future study.

\section{ACKNOWLEDGMENT}

The authors thank the anonymous reviewer for bringing the work of Johnson et al. to their attention.

\section{REFERENCES}

[1] J. M. T. Thompson and G. W. Hunt, Elastic Instability Phenomena. New York: Wiley, 1984, ch. 4.

[2] J. M. T. Thompson, Instabilities and Catastrophes in Science and Engineering. New York: Wiley, 1982, ch. 2.

[3] T. Schioler and S. Pellegrino, "Multi-configuration space frames," presented at the 45th AIAA/ASME/ASCE/AHS/ASC Structures, Structural Dynamics, and Materials Conf., Palm Springs, CA, Apr. 19-22, 2004, Paper AIAA 2004-1529.

[4] E. Chater, J. W. Hutchinson, and K. W. Neale, "Buckle propagation on a beam on a nonlinear elastic foundation," in Collapse: The Buckling of Structures in Theory and Practice, J. M. T. Thompson and G. W. Hunt, Eds. Cambridge, U.K.: Cambridge Univ. Press, 1982, pp. 31-41.

[5] Y. C. Fung and A. Kaplan, "Buckling of low arches or curved beams of small curvatures," NACA Technical Note 2840, 1952.

[6] J. S. Humphreys, "On dynamic snap buckling of shallow arches," AIAA J., vol. 4, no. 5, pp. 878-886, 1966.

[7] M. H. Lock, "Snapping of a shallow sinusoidal arch under a step pressure load," AIAA J., vol. 4, no. 7, pp. 1249-1256, 1966.

[8] W. Nachbar and N. C. Huang, "Dynamic snap-through of a simple viscoelastic truss," in Q. Appl. Math., vol. 25, Apr. 1967, pp. 65-82.

[9] C. S. Hsu, "The effect of various parameters on the dynamic stability of a shallow arch," J. Appl. Mech., vol. 34, no. 2, pp. 349-358, 1967.

[10] C. S. Hsu, "Stability of shallow arches against snap-through under timewise step loads," J. Appl. Mech., vol. 35, no. 1, pp. 31-39, Mar. 1968.

[11] C. S. Hsu, "Equilibrium configurations of a shallow arch of arbitrary shape and their dynamic stability character," Int. J. Non-Linear Mech., vol. 3, no. 2, pp. 113-136, Jun. 1968.

[12] N. C. Huang and W. Nachbar, "Dynamic snap-through of imperfect viscoelastic shallow arches," J. Appl. Mech., vol. 35, no. 2, pp. 289-296, 1968.

[13] A. B. Pippard, "The elastic arch and its modes of instability," Eur. J. Phys., vol. 11 , no. 6, pp. 359-365, Nov. 1990 .

[14] P. Patrício, M. Adda-Bedia, and M. Ben Amar, "An elastica problem: Instabilities of an elastic arch," Physica D, vol. 124, no. 1-3, pp. 285295, 1998. 
[15] A. Boudaoud, P. Patrício, Y. Couder, and M. Ben Amar, "Dynamics of singularities in a constrained elastic plate," Nature, vol. 407, no. 6805, pp. $718-720$, Oct. 2000.

[16] M. Vangbo, "An analytical analysis of a compressed bistable buckled beam," Sens. Actuators A, Phys., vol. 69, no. 3, pp. 212-216, 1998.

[17] C. Goll, W. Bacher, B. Büstgens, D. Maas, W. Menz, and W. K. Schomburg, "Microvalves with bistable buckled polymer diaphragms," J. Micromech. Microeng., vol. 6, no. 1, pp. 77-79, Mar. 1996.

[18] V. D. Kugel, B. Xu, M. Zhang, and L. E. Cross, "Bimorph-based piezoelectric air acoustic transducer: Model," Sens. Actuators A, Phys., vol. 69, no. 3, pp. 234-242, Sep. 1998.

[19] M. Vangbo and Y. Bäcklund, "A lateral symmetrically bistable buckled beam," J. Micromech. Microeng., vol. 8, no. 1, pp. 29-32, Mar. 1998.

[20] M. T. Saif, "On a tunable bistable MEMS - Theory and experiment," J. Microelectromech. Syst., vol. 9, no. 2, pp. 157-170, Jun. 2000.

[21] M. Sulfridge, M. T. Saif, N. Miller, and K. O'Hara, "Optical actuation of a bistable MEMS," J. Microelectromech. Syst., vol. 11, no. 5, pp. 574-583, Oct. 2002.

[22] J. Qiu, J. H. Lang, and A. H. Slocum, "A curved-beam bistable mechanism," J. Microelectromech. Syst., vol. 13, no. 2, pp. 137-146, Apr. 2004.

[23] I. Hwang, Y. Shim, and J. Lee, "Modeling and experimental characterization of the chevron-type bi-stable microactuator," J. Micromech. Microeng., vol. 13, no. 6, pp. 948-954, Nov. 2003.

[24] J. S. Ko, M. L. Lee, D. Lee, C. A. Choi, and Y. T. Kim, "Development and application of a laterally driven electromagnetic microactuator," Appl. Phys. Lett., vol. 81, no. 3, pp. 547-549, Jul. 2002.

[25] P. M. Osterberg and S. D. Senturia, "M-TEST: A test chip for MEMS material property measurement using electrostatically actuated test structures," J. Microelectromech. Syst., vol. 6, no. 2, pp. 107-118, Jun. 1997.

[26] B. Choi and E. G. Lovell, "Improved analysis of microbeams under mechanical and electrostatic loads," J. Micromech. Microeng., vol. 7, no. 1, pp. 24-29, Mar. 1997.

[27] E. S. Hung and S. D. Senturia, "Extending the travel range of analog-tuned electrostatic actuators," J. Microelectromech. Syst., vol. 8, no. 4, pp. 497505, Dec. 1999

[28] O. Bochobza-Degani and Y. Nemirovsky, "Modeling the pull-in parameters of electrostatic actuators with a novel lumped two degrees of freedom pull-in model," Sens. Actuators A, Phys., vol. 97/98, pp. 569-578, Apr. 2002.

[29] E. M. Abdel-Rahman, M. I. Younis, and A. H. Nayfeh, "Characterization of the mechanical behavior of an electrically actuated microbeam," J. Micromech. Microeng., vol. 12, no. 6, pp. 759-766, Nov. 2002.

[30] G. W. Wang, Y. Zhang, Y. P. Zhao, and G. T. Yang, "Pull-in instability study of carbon nanotube tweezers under the influence of van der Waals forces," J. Micromech. Microeng., vol. 14, no. 8, pp. 1119-1125, Aug. 2004.

[31] Y. Zhang and Y. P. Zhao, "Numerical and analytical study on the pull-in instability of micro-structure under electrostatic loading," Sens. Actuators A, Phys., vol. 127, no. 2, pp. 366-380, 2006.

[32] W. Fang and J. A. Wickert, "Post buckling of micromachined beams," J. Micromech. Microeng., vol. 4, no. 3, pp. 116-122, Sep. 1994.

[33] M. Chiao and L. Lin, "Self-buckling of micromachined beams under resistive heating," J. Microelectromech. Syst., vol. 9, no. 1, pp. 146-151, Mar. 2000.

[34] W. Fang and J. A. Wickert, "Determining mean and gradient residual stresses in thin films using micromachined cantilevers," J. Micromech. Microeng., vol. 6, no. 3, pp. 301-309, 1996.

[35] E. R. Johnson, M. W. Hyer, and D. M. Carper, "Response of composite material shallow arch to concentrated load," J. Aircraft, vol. 23, no. 6, pp. 529-536, 1986.

[36] G. A. Korn and T. M. Korn, Mathematical Handbook for Scientist and Engineers, 2nd ed. New York: McGraw-Hill, 1968.

[37] C. H. Mastrangelo and C. H. Hsu, "Mechanical stability and adhesion of microstructures under capillary forces-Part I: Basic theory," J. Microelectromech. Syst., vol. 2, no. 1, pp. 33-43, Mar. 1993.

[38] J. E. Sader and C. P. Green, "In-plane deformation of cantilever plates with applications to lateral force microscopy," Rev. Sci. Instrum., vol. 75, no. 4, pp. 878-883, Apr. 2004.

[39] T. Chang and R. R. Craig, Jr., "Normal modes of uniform beam," J. Eng. Mech. Div., ASCE, vol. 95, no. EM 4, pp. 1027-1031, 1969.

[40] W. H. Press, S. A. Teukolsky, W. T. Vetterling, and B. P. Flannery, Numerical Recipes, 2nd ed. Cambridge, U.K.: Cambridge Univ. Press, ch. 9.

[41] Z. Xiao, M. Chen, G. Wu, C. Zhao, D. Zhang, Y. Hao, G. Zhang, and $\mathrm{Z}$. Li, "Silicon micro-accelerometer with $\mathrm{mg}$ resolution, high linearity and large frequency bandwidth fabricated with two mask bulk process," Sens. Actuators A, Phys., vol. 77, no. 2, pp. 113-119, Oct. 1999.
[42] Z. Li, Z. Xiao, Y. Hao, G. Wu, and Y. Wang, "A bulk micromachined vibratory lateral gyroscope fabricated with wafer bonding and deep trench etching," Sens. Actuators A, Phys., vol. 83, no. 1, pp. 24-29, May 2000.

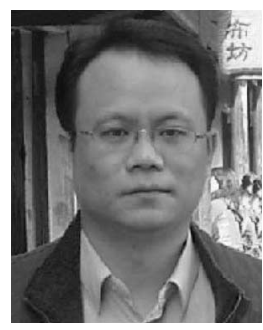

Yin Zhang received the B.S. degree from the Department of Mechanics, Peking University, Beijing, China, in 1997, and the Ph.D. degree from the Department of Mechanical Engineering, University of Connecticut, Storrs, in 2003.

From 2003 to 2004, he was an Assistant Professor in the State Key Laboratory of Nonlinear Mechanics, Institute of Mechanics, Chinese Academy of Sciences, Beijing, where he has been an Associate Professor since 2004. His current research interests include MEMS/NEMS structural mechanics, dynamics, and interface properties.

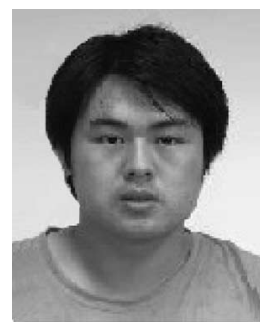

Yisong Wang received the B.S. degree from the Department of Microelectronics, Nankai University, Tianjin, China, in 2004. He is currently working toward a graduate degree at the National Key Laboratory of Nano/Micro Fabrication Technology, Institute of Microelectronics, Peking University, Beijing, China.

His research interests include semiconductor process modeling and RF MEMS.

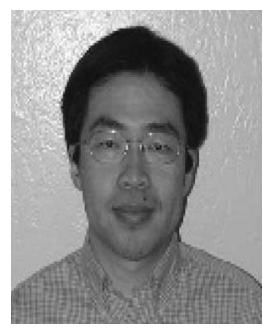

Zhihong Li (M'02) received the B.S. degree from the Department of Computer Science and Technology, Peking University, Beijing, China, in 1992, and the $\mathrm{Ph} . \mathrm{D}$. degree from the Institute of Microelectronics, Peking University, majoring in VLSI technology and reliability, in 1997.

After receiving the Ph.D. degree, he joined the MEMS Group, Institute of Microelectronics, Peking University. From 2000 to 2004, he was a Postdoctoral Researcher at Cornell University, Ithaca, NY, and the University of California, Davis $\mathrm{He}$ is currently a Professor and the Director of the MEMS Center, Institute of Microelectronics, Peking University. His research interests include design and fabrication of microelectromechanical systems (MEMS), in particular, RF MEMS and Bio MEMS.

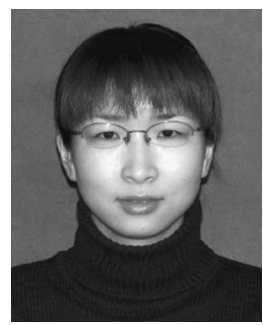

Yubo Huang is currently working toward the Ph.D. degree in measurement technology and instruments in the College of Precision Instrument and OptoElectronics Engineering, Tianjin University, Tianjin, China.

In 2004, she joined the State Key Laboratory of Precision Measuring Technology and Instruments, Tianjin University. Her research focuses on optic measurement and analysis of mechanical properties of MEMS.

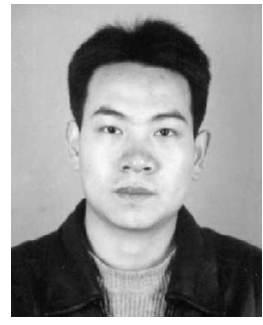

Dachao $\mathbf{L i}$ received the B.S. degree from the Department of Precision Instruments, Tianjin University, Tianjin, China, in 1998, and the Ph.D. degree in MEMS technology from the College of Precision Instruments and Opto-Electronics Engineering, Tianjin University, in 2003.

After receiving the Ph.D. degree, he joined the Micro/Nanomeasurement Group. From 2004 to 2006, he was a Postdoctoral Researcher at Peking University. He is currently an Associate Professor in the College of Precision Instruments and OptoElectronics Engineering, Tianjin University. His research interests include micro/nano metrology and design and fabrication of microelectromechanical systems (MEMS). 